\title{
Bacterial Gastritis Aggravates Atherosclerosis
}

\section{Richard M Fleming ${ }^{1 *}$, Matthew R Fleming ${ }^{1}$ and Tapan K. Chaudhuri ${ }^{2}$}

${ }^{1}$ FHHI-OmnificImaging-Camelot, Los Angeles, CA, USA

${ }^{2}$ Eastern Virginia Medical School, Norfolk, Virginia

*Corresponding Author: Richard M Fleming, FHHI-OmnificImaging-Camelot, Los Angeles, CA, USA.

Received: September 05, 2019; Published: September 13, 2019

DOI: $10.31080 /$ ASPS.2019.03.0399

This case represents a 29-year-old Caucasian male with a history of gastritis, who developed retrosternal discomfort with exertion over several weeks. The discomfort was associated with nausea.

His fasting lipoprotein analysis revealed a total cholesterol of 201, LDLc of 137, HDL of 39 and triglyceride level of 123. The TC/ HDLc ratio was ated 5.2. Additional blood tests showed an elevated C-RP of 5.2 (nl: <0.5) with acute phase antibodies for H. Pylori [3-5].

He underwent pharmacologic stress myocardial perfusion imaging (MPI) with a technetium-99m based agent $[1,2]$.

Following MPI he underwent cardiac catheterization, with results shown in Figure 2.

The patient underwent antibiotic treatment for H. Pylori, with resolution of angina, a normalization of C-RP and improved MPI as shown in Figure 3.

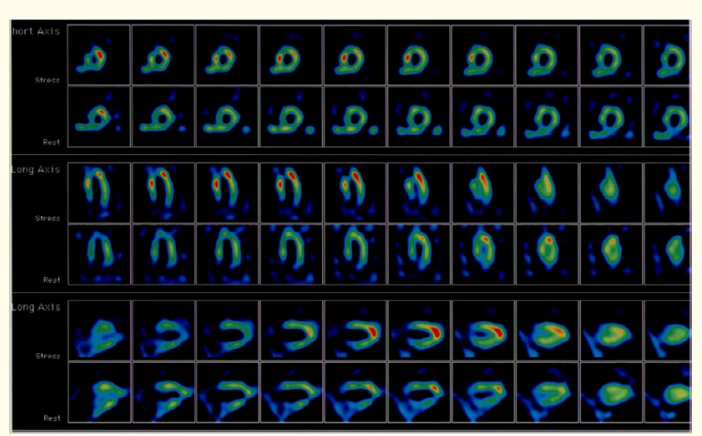

Figure 1: Pre-treatment myocardial perfusion imaging.

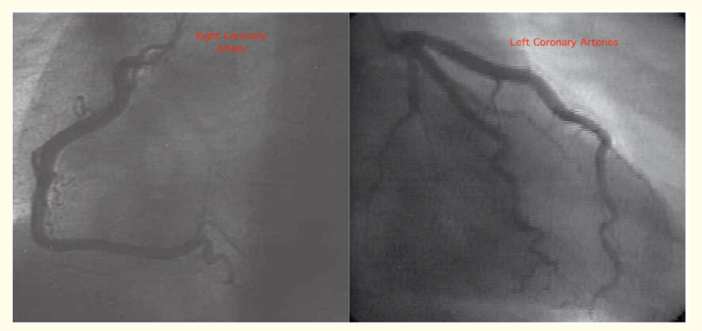

Figure 2: Coronary arteriogram.

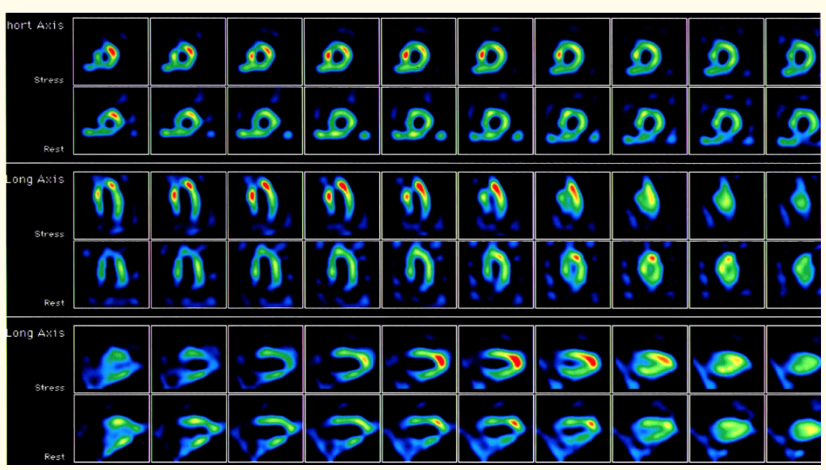

Figure 3: Post-treatment myocardial perfusion imaging.

\section{Acknowledgment}

All figures reproduced with expressed consent of first author.

\section{Questions}

What percentage of acute myocardial infarctions present with a prior angiogram with less than $30 \%$ diameter narrowing? (the answer is $\mathrm{d}-70 \%$ )
a) $10 \%$
b) $20 \%$
c) $50 \%$
d) $70 \%$
e) $90 \%$

Which of the following is a marker of an acute inflammatory process? (the answer is d-all of the above).
a) C-RP
b) Fibrinogen
c) Interluekin-6
d) All of the above

If the angiogram reveals no evidence of stenosis, then

a) There is no coronary artery disease present

b) Coronary artery disease may be present, but coronary lumen disease is absent. 
c) Other causes of chest pain should be looked for, because the angiogram is the "gold standard" for detecting heart disease

d) The patient should undergo a treadmill study. (the answer is b-the angiogram is useful for detecting focal stenotic disease, but does not show diffuse disease, medium or small blood vessel disease, or endothelial dysfunction).

Volume 3 Issue 10 October 2019

(c) All rights are reserved by Richard M Fleming., et al. 